\title{
Evaluation of re-hospitalized COVID-19 patients in a hospital
}

\section{SUMMARY}

PURPOSE: This study intends to investigate the reasons for re-hospitalization, complaints, and prognoses of COVID-19 patients after being discharged.

METHODS: COVID-19 patients who were re-hospitalized at the Sakarya University Training and Research Hospital were examined. Reverse transcriptase-polymerase chain reaction (RT-PCR), tomography and laboratory results, demographic characteristics, and prognostic results were recorded retrospectively.

RESULTS: A total of 60 patients, including 26 males (43.3\%) and 34 females (56.7\%), with repeated admissions to the hospital for COVID-19 symptoms, were included in the study with a mean age of 56.9 ( \pm 22.5$)$ (median value $=61$, age range $=3-88$ ). The number of days of the second hospitalization was statistically significantly higher $(p<0.05)$. Patient age and number of days of hospitalization were strongly positively correlated $(p<0.01)$. A total of 11 patients $(18 \%)$ had negative results in their first RT-PCR and subsequently tested positive in their second hospitalization. In addition, $10(17.5 \%)$ of the patients who underwent thoracic tomography had unilateral involvement, 34 (59.6\%) had bilateral involvement, and $13(22.8 \%)$ had no significant results. Note that 4 (6.6\%) of the patients re-hospitalized died in the hospital, while 56 (93.4\%) were discharged once more. All of the four patients that died were female with a mean age of 81.5 years.

CONCLUSION: Particularly patients with advanced age and comorbidities should be examined more carefully when discharged; if their complaints are repeated, they should be advised to quickly contact the emergency service.

KEYWORDS: COVID-19, re-hospitalization, coronavirus

\section{INTRODUCTION}

The disease caused by the SARS-CoV-2 virus, which is part of the family of Coronaviruses, has been named COVID-19 ${ }^{1}$. After being infected by the SARS-CoV-2 virus, the average incubation period is reported to be of 6.4 days (2.1-11.1 days) ${ }^{2}$. COVID-19 has a wide range of clinical manifestations, from symptoms such as asymptomatic disease and mild upper respiratory tract infection to severe pneumonia accompanied by respiratory failure, which might subsequently result in death ${ }^{3} 2019$, Wuhan, China, 
has experienced an outbreak of coronavirus disease 2019 (COVID-19. Some studies report hospitalizations of 19 days, while the average length of hospitalizations varies depending on the country ${ }^{4}$. There are healthcare centers that suggest patient discharge after ensuring that the patient meets criteria such as improvement in symptoms, improvement in check-up thoracic tomography, testing negative in at least two RT-PCR tests with an interval of $24 \mathrm{~h}$, and having no fever for at least three days ${ }^{3} 2019$, Wuhan, China, has experienced an outbreak of coronavirus disease 2019 (COVID-19. Patients who are discharged from the hospital might then be admitted asymptomatically or symptomatically and require re-hospitalization ${ }^{5}$. This study intends to help clinical physicians estimate which COVID-19 patients might have a higher potential of re-hospitalization.

\section{METHODS}

The Sakarya University Training and Research Hospital (SUEAH) is a 1000-bed, fully equipped tertiary public hospital located in the Sakarya Province of Turkey and has served as a pandemic hospital since March 03, 2020.

After obtaining written permission from the chief physician's office, the patient data were collected through retrospective reviewing of patient files and the hospital information system covering between March 16 and May 8, 2020.

Thoracic tomography was then performed using Toshiba Alexion 16 tomography scanner as a low-dose non-contrast tomography.

Inclusion criteria: Re-hospitalized RT-PCR-positive COVID-19 patients and the hospital records of all age groups were examined and included in the study.

Exclusion criteria: Those re-hospitalized for reasons other than COVID-19, despite testing positive in the RT-PCR test or negative in the RT-PCR test while their tomography findings supported COVID-19 disease were excluded from the study.

Statistical analysis: The data obtained were statistically analyzed using SPSS 21 . The Chi-square test was used to compare categorical data, and $\mathrm{p}<0.05$ was considered to be statistically significant. The Kolmogorov-Smirnov test was used to assess the normality of the data in terms of distribution. For the statistical assessment of non-normally distributed data, nonparametric tests were used. The Mann-Whitney U test was then used to compare two independent variables, while the Kruskal-Wallis $\mathrm{H}$ test was used to compare more than two independent variables; $\mathrm{p}<0.05$ was accepted to be statistically significant.

\section{RESULTS}

The number of patients re-hospitalized was 60; 26 of them $(43.3 \%)$ were male and 34 (56.7\%) were female. The mean age of the patients was $56.9( \pm$ $22.5)$ and the age range was $3-88$ years. Moreover, the mean length of the initial hospitalization was 4.6 days ( \pm 2.87 days). The mean time until re-hospitalization after discharge was 6.8 days $( \pm 4.75)$; however, no correlation was found between the time until re-hospitalization after discharge and the second hospitalization $(p>0.05)$. The patients had a mean length of the second hospitalization of 7.4 days $( \pm 6.8)$ and the duration of the stay was between 1 and 36 days. There was a moderate, positive, statistically significant correlation between the number of days of the first hospitalization and the number of days of the second hospitalization $(p<0.05)$. Accordingly, compared to the first hospitalization, the second one was longer in 34 patients (56.7\%), shorter in 17 patients $(28.3 \%)$, and the same in 9 patients (15\%). The patients were then divided into three age groups, i.e., 0-17 years, 18-64 years, and $>65$ years. After analyzing the correlation between the age groups and their hospitalizations, there was a significant difference between the first hospitalization, the second hospitalization, and the total length of hospitalization $(p<0.05)$. According to the post-hoc analysis, there was a significant difference between the group of 0-17 years and the groups of 18-64 years and of $>65$ years in terms of second hospitalization $(p<0.05)$. Moreover, there was no significant difference in the age group of 18-64 years and the group of $>65$ years in terms of second hospitalization and the length of hospitalization $(p>0.05)$. Nevertheless, there was no significant correlation between patient age and the time until re-hospitalization after discharge $(\mathrm{p}>0.05)$.

Note that $49(81.7 \%)$ of these patients tested positive, and 11 (18.3\%) tested negative in the RT-PCR tests during their first hospitalization. Of the 49 patients who tested positive in the RT-PCR tests, 22 were not tested again, 17 tested negative, and 10 tested positive again during their second hospitalization. The first RT-PCR result was negative in 11 patients (18\%), who subsequently tested positive in 
their second hospitalization. Of the total number of patients, 57 (95\%) had CT. Note that for 17 patients (28.3\%), CT was performed only during their first admission; for 39 patients (65\%), it was during both first and second hospitalizations; and for one patient $(1.7 \%)$ it was performed during their second admission. Of the patients who underwent thorax CT, 10 patients (17.5\%) had unilateral involvement, 34 (59.6\%) had bilateral involvement, and 13 (22.8\%) had no significant findings. There was a significant difference between those without significant CT findings and those with bilateral involvement in terms of initial hospitalization $(\mathrm{p}<0.05)$.

Note that $4(6.6 \%)$ of the re-hospitalized patients died in the hospital, and all of them were female. They had an age range of 73-86 years and a mean age of 81.5 years and bilateral involvement in their thoracic CT. They had an initial hospitalization range of 3-10 days and their mean initial hospitalization length was 6 days. Moreover, three of them were re-hospitalized for impaired consciousness and syncope and 1 for cough and fever 1-7 days after their first discharge. All of these patients had at least two (2-6) concomitant chronic diseases. Three of the patients died on the seventh day of their second hospitalization and one died on the ninth day of the second hospitalization.

Of the re-hospitalized patients, 23 (38.3\%) had cough, 20 (33.3\%) dyspnea, 13 (21.6\%) fever, while 10 $(20 \%)$ had no symptoms at all. The symptoms of the patients are listed in Table 1.

When examining the comorbidity status of the patients re-hospitalized, 19 (31.7\%) did not have any

TABLE 1. SYMPTOMS OF RE-HOSPITALIZED PATIENTS

\begin{tabular}{l|l|l} 
Symptom & Patient Count & Percent \% \\
\hline Cough & 23 & 38.3 \\
\hline Dyspnea & 20 & 33.3 \\
\hline Fever & 13 & 21.7 \\
\hline Weakness & 10 & 16.7 \\
\hline Asymptomatic & 10 & 16.7 \\
\hline Syncope & 5 & 8.3 \\
\hline Chest pain & 5 & 8.3 \\
\hline Back pain & 4 & 6.7 \\
\hline Diarrhea & 4 & 6.7 \\
\hline Sore throat & 3 & 5 \\
\hline Stomachache & 3 & 5 \\
\hline Anosmia & 2 & 3.3 \\
\hline Nausea & 2 & 3.3 \\
\hline Headache & 1 & 1.7
\end{tabular}

TABLE 2. NUMBER OF COMORBIDITIES OF REHOSPITALIZED COVID-19 PATIENTS

\begin{tabular}{l|l|l} 
Number of Comorbidities & \multicolumn{1}{l}{ Number of Patients } & Percent \% \\
\hline 0 & 19 & 31.7 \\
\hline 1 & 7 & 11.7 \\
\hline 2 & 11 & 18.3 \\
\hline 3 & 14 & 23.3 \\
\hline 4 & 1 & 1.7 \\
\hline 5 & 6 & 10 \\
\hline 6 & 2 & 3.3 \\
\hline
\end{tabular}

TABLE 3. TYPES OF COMORBIDITIES OF REHOSPITALIZED COVID-19 PATIENTS

\begin{tabular}{l|l|l}
\hline Comorbidity & Number of Patients & Percent \% \\
\hline Hypertension & 32 & 53.3 \\
\hline Cardiovascular diseases & 19 & 31.7 \\
\hline Diabetes & 16 & 26.7 \\
\hline Cerebrovascular diseases & 11 & 18.3 \\
\hline Psychiatric diseases & 10 & 16.7 \\
\hline Chronic lung diseases & 9 & 15 \\
\hline Cancer & 4 & 6.7 \\
\hline Chronic renal failure & 3 & 5 \\
\hline Immunosuppressive states & 1 & 1.7 \\
\hline
\end{tabular}

chronic disease, 14 (23.3\%) had three comorbidities, and 11 (18.3\%) had two comorbidities. Table 2 lists the number of comorbidities. There was a statistically significant correlation between the presence of comorbidity and the number of days of the first hospitalization and the total number of hospitalization days $(p<0.05)$. The initial hospitalization of patients with comorbidities was longer than those without comorbidities. There was a positive correlation of statistically moderate significance between the number of concomitant chronic diseases and the number of days of the first and second hospitalization $(p<0.05)$. As for comorbidities, 32 patients (53.3\%) had hypertension, 19 (31.7\%) had cardiovascular diseases (CVH), and 16 (26.7\%) had diabetes. The comorbid diseases of the patients are shown in Table 3.

After comparing the values of lactate, ferritin, d-Dimer, white blood cell (WBC), platelets (PLT), lymphocyte (LYM), lactate dehydrogenase (LDH), urea, creatinine, aspartate aminotransferase (AST), alanine aminotransferase (ALT), and C-reactive protein (CRP) during the first and second hospitalization, there was a statistically significant difference in lactate, ferritin, d-Dimer, platelet, CRP, and LDH $(p<0.05)$. Table 4 lists the evaluation of the laboratory results. 


\section{DISCUSSION}

There is only a limited volume of information about COVID-19 patients who are re-hospitalized after discharge. Chen et al. reported that 11 patients were re-hospitalized, and Peng et al. reported that 7 patients were re-hospitalized after discharge ${ }^{5,6}$. In a prospective study conducted by Wang et al., 5 (3.82\%) of 13 COVID-19 patients discharged were re-hospitalized ${ }^{7}$ distribution of quarantine locations, and the infection status of the contacts of COVID-19 patients after discharge. $\backslash n \backslash n \backslash n$ Design $\backslash n$ A prospective cohort study $\backslash n$ In \n Methods \n Demographics, baseline characteristics of 131 COVID-19 patients discharged from February 3 to 21, 2020 in Wuhan, China were collected and analyzed by reviewing the medical records retrospectively. Post-hospitalization data related to clinical outcomes, quarantine locations and close contact history were obtained by following up the patients every week up to 4 weeks. In $\backslash n \backslash n$ Results $\backslash n 53$ (40.05\%. Moreover, Wang et al. reported that two patients discharged after recovering from pneumonia were re-hospitalized because of intestinal infections caused by Sars-Cov- $2^{8}$. Cao et al. reported that 8 out of 108 patients who were discharged between February 10 and April 13, 2020, were re-hospitalized ${ }^{9}$. Richardson et al. examined 5700 patients who were hospitalized for COVID-19 in New York City and reported that 45 patients $(2.2 \%)$ were re-hospitalized ${ }^{10}$. In our study, the number of patients who were admitted to the hospital for different reasons after discharge is 60 .

In the study of Safiya et al., ${ }^{22}$ (48.8\%) of the re-hospitalized COVID-19 patients were aged $>65$ years, and $22(48.8 \%)$ were aged between 18 and 64 years $^{10}$. The mean age of patients was 48.4 years in the study by Chen et al., ${ }^{54} .3$ years in the study by Cao et al., and 48.7 years in the study by XingyuWang et al. ${ }^{6,9,11}$. The mean age of the patients included in our study is 56.9. Based on these results, the mean age of the patients in our study is older than that of the patients in other studies. Chen et al. reported that re-admitted patients were re-hospitalized $16 \pm 7.14$ days after discharge, while Wang et al. reported that five patients were re-hospitalized within 1-2 weeks and 3 patients within 3-4 weeks ${ }^{6,11}$. Safiya et al. reported that 45 discharged patients were re-hospitalized on an average of 3 days after discharge, while Wang et al. reported that two patients were re-hospitalized one day after discharge ${ }^{8,10}$. In our study, the mean time until re-hospitalization after discharge is $6.82 \pm 4.75$ days, which is shorter than that reported by Chen et al. and longer than that reported by Safiya et al. and Wang et al. Although Chen et al. and Peng et al. reported that there was a shortening in the lengths of second hospitalizations ${ }^{5,6}$, $56.7 \%$ of the patients had longer durations in the second hospitalization, and these results did not coincide with the results of Chen et al. To better understand the cause of this noncoincidence, it may be useful to examine the reasons for patient hospitalizations. In most of the abovementioned studies that cover information about re-hospitalization, the RT-PCR tests were negative when patients were discharged. In this case, it is observed that the eight patients that Cao et al. included in their study had no symptoms but were re-hospitalized only because their RT-PCR test came back positive 9 . In support of this study, Peng et al. reported seven re-hospitalized patients, and five of these patients were re-hospitalized despite having no symptoms and only because their RT-PCR test results came back positive or they had abnormalities in their tomography images ${ }^{5}$. However, all of the 11 patients that Chen et al. included in their study had at least one of the complaints of cough (54.5\%), fever (27.3\%), or malaise (27.3\%) ${ }^{6}$. In our study, 10 patients $(16.6 \%)$ were re-hospitalized based on tomography findings and RT-PCR test results, although they were asymptomatic during the second admission, while 50 patients (83.4\%) had one or several complaints. The top three symptoms include cough in 23 patients (38.3\%), dyspnea in 20 patients (33.3\%), and fever in 13 patients (21.7\%). Moreover, severe symptoms such as syncope (8.3\%) and chest pain (8.3\%) were observed in some patients. Complaints during the second admission include more serious symptoms such as dyspnea, syncope, and chest pain, which may be considered to affect the higher number of days of hospitalization in the second admission. The mean hospitalization of 10 asymptomatic patients in the first hospitalization was 4.8 days; however, on average, this period was reduced to 3.3 days in the second admission. Nevertheless, the mean duration of the first hospitalization in patients with one or more symptoms was 4.5 days, while the mean number of days in the second hospitalization increased to 8.26 days. However, our study does not coincide with the study by Chen et al. since the hospitalization of the symptomatic patients included in our study increased in their second admission. Moreover, as mentioned above, the mean age of our patients is higher than that of patients in other studies, which may affect the longer duration of their second hospitalization. In other studies, limited information was 
given about the mortality of re-hospitalized patients. Of the patients included in our study, four (6.6\%) died in the hospital during their second hospitalization.

Note that there is no sufficient information in the literature concerning the comorbidities of re-hospitalized patients. In 19 (31.6\%) of the patients included in our study, there were no additional diseases, while 41 patients (68.4\%) had at least one concomitant disease. According to a meta-analysis investigating comorbidities of COVID-19 patients, the four most common comorbidities are as follows: hypertension (14\%-22\%), diabetes $(6 \%-11 \%)$, cardiovascular diseases $(4 \%-7 \%)$, and chronic lung diseases (1\%-3\%), respectively ${ }^{12}$. The most common comorbidities of the patients in our study include hypertension, cardiovascular diseases, diabetes, and cerebrovascular events. The concomitant diseases of the patients in our study are similar to the comorbidities of overall COVID-19 patients.

\section{CONCLUSION}

Re-hospitalized patients may have at least one additional disease, there might not be very specific symptoms during the second admission, and comorbidities may increase mortality especially in elderly women when they are re-admitted with serious symptoms such as syncope. When being discharged, such patients can be advised to be more careful and immediately contact the hospital on any negative development. For asymptomatic patients, a more detailed assessment of their second hospitalization may help reduce unnecessary hospitalization.

\section{Permission}

Obtained from Sakarya University Training and Research Hospital Administration on 01/04/2020.

\section{Conflict}

There is no conflict of interest regarding the article. No financial aid has been received from any institution or organization.

\section{Author's Contribution}

Fatih GUNEYSU -visualization, resources, editing. Ensar DURMUS - data curation, formal analysis, methodology, writing.

\section{RESUMO}

OBJETIVO: Este estudo pretende investigar as causas para re-hospitalizações, as reclamações e os prognósticos de pacientes com COVID-19 após a alta hospitalar.

MÉTODOS: Pacientes com COVID-19 internados que foram re-hospitalizados no Sakarya University Training and Research Hospital foram examinados. Os resultados da reação em cadeia de polimerase precedida de transcrição reversa (RT-PCR), tomografia e dos exames laboratoriais, as características demográficas e os resultados prognósticos foram registrados retrospectivamente.

RESULTADOS: Um total de 60 pacientes, 26 do sexo masculino (43,3\%) e 34 do sexo feminino (56,7\%), com internações repetidas devido a sintomas de COVID-19 foram incluídos no estudo, com uma idade média de 56,9 ( $\pm 22,5)$ (mediana = 61, faixa etária = 3-88). O número de dias da segunda internação foi estatisticamente significativamente maior $(p<0,05)$. A idade do paciente e o número de dias de internação apresentaram uma forte correlação positiva $(p<0,01)$. Um total de 11 pacientes (18\%) apresentaram resultados negativos no primeiro RT-PCR e posteriormente tiveram resultados positivos na segunda internação. Além disso, 10 (17,5\%) dos pacientes submetidos a tomografia de tórax apresentaram envolvimento unilateral, 34 (59,6\%) bilateral, e 13 (22,8\%) não apresentaram resultados significativos. Nota-se que 4 (6,6\%) dos pacientes re-hospitalizados morreram no hospital, enquanto $56(93,4 \%)$ receberam alta mais uma vez. Todos os quatro pacientes que morreram eram do sexo feminino, com idade média de 81,5 anos.

CONCLUSÃO: Principalmente pacientes com idade avançada e comorbidades devem ser examinados com mais cuidado no momento da alta hospitalar; caso suas queixas se repitam, eles devem ser aconselhados a contatar o serviço de emergência o quanto antes.

PALAVRAS-CHAVE: COVID-19, re-hospitalização, coronavírus

\section{REFERENCES}

1. Huang C, Wang Y, Li X, Ren L, Zhao J, Hu Y, et al. Clinical features of patients infected with 2019 novel coronavirus in Wuhan, China. The Lancet. 2020 Feb;395(10223):497-506.

2. Lai C-C, Shih T-P, Ko W-C, Tang H-J, Hsueh P-R. Severe acute respiratory syndrome coronavirus 2 (SARS-CoV-2) and coronavirus disease-2019
(COVID-19): The epidemic and the challenges. Int J Antimicrob Agents. 2020 Mar;55(3):105924.

3. Zhou F, Yu T, Du R, Fan G, Liu Y, Liu Z, et al. Clinical course and risk factors for mortality of adult inpatients with COVID-19 in Wuhan, China: a retrospective cohort study. The Lancet. Elsevier; 2020 Mar 28;395(10229):10541062. PMID: 32171076 
4. Wang zhuo, Ji IS, Liu Y, Liu R, Zha Y, Chang X, et al. Survival analysis of hospital length of stay of novel coronavirus (COVID-19) pneumonia patients in Sichuan, China [Internet]. Health Policy; 2020 Apr. Available from: http:// medrxiv.org/lookup/doi/10.1101/2020.04.07.20057299

5. Peng J, Wang M, Zhang G, Lu E. Seven discharged patients turning positive again for SARS-CoV-2 on quantitative RT-PCR. Am J Infect Control [Internet]. 2020 Apr 10 [cited 2020 Apr 21]; Available from: https://www.ncbi.nlm.nih.gov/pmc/articles/PMC7151314/ PMCID: PMC7151314

6. Chen M, An W, Xia F, Yang P, Li K, Zhou Q, et al. Clinical Characteristics of Re-hospitalized Patients with COVID-19 in China. J Med Virol. 2020 May 13;jmv.26002

7. Wang $X, X u H$, liang $H$, Wang L, Lu C, Wei X, et al. The Clinical Features and Outcomes of Discharged Coronavirus Disease 2019 Patients-A Prospective Cohort Study. QJM Int ] Med. 2020 May 22;hcaa178.
8. Wang $X$, Zhou Y, liang N, Zhou O, Ma W-L. Persistence of intestinal SARSCoV-2 infection in patients with COVID-19 leads to re-admission after pneumonia resolved. Int | Infect Dis. 2020 Jun;95:433-435.

9. Cao H, Ruan L, Liu J, Liao W. The clinical characteristic of eight patients of COVID-19 with positive RT-PCR test after discharge. | Med Virol. 2020 May 15;jmv.26017.

10. Richardson S, Hirsch JS, Narasimhan M, Crawford JM, McGinn T, Davidson $\mathrm{KW}$, et al. Presenting Characteristics, Comorbidities, and Outcomes Among 5700 Patients Hospitalized With COVID-19 in the New York City Area. JAMA. 2020 May 26;323(20):2052.

11. Wang $X, X u H$, liang $H$, Wang $L$, Lu $C$, Wei $X$, et al. The Clinical Features and Outcomes of Discharged Coronavirus Disease 2019 Patients-A Prospective Cohort Study. QJM Int | Med. 2020 May 22;hcaa178.

12. Yang J, Zheng Y, Gou X, Pu K, Chen Z, Guo Q, et al. Prevalence of comorbidities and its effects in patients infected with SARS-CoV-2: a systematic review and meta-analysis. Int J Infect Dis. 2020 May;94:91-95. 